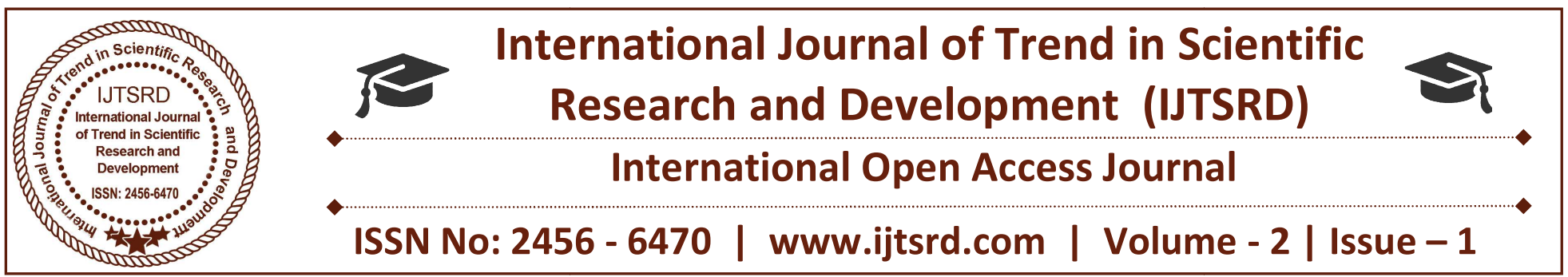

\title{
Diagnosis of hyperglycemia using Artificial Neural Networks
}

\author{
Abid Sarwar \\ Department of Computer Science \& IT (Bhaderwah Campus) \\ University of Jammu, Jammu, India
}

\section{ABSTRACT}

The aim of Artificial Intelligence is to develop the machines to perform the tasks in a better way than the humans. Another aim of Artificial Intelligence is to understand the actions whether it occurs in humans, machines or animals. As a result, Artificial Intelligence is gaining Importance in science and engineering fields. The use of Artificial Intelligence in medical diagnosis too is becoming increasingly common and has been used widely in the diagnosis of cancers, tumors, hepatitis, lung diseases, etc... The main aim of this paper is to build an Artificial Intelligent System that after analysis of certain parameters can predict that whether a person is diabetic or not. Diabetes is the name used to describe a metabolic condition of having higher than normal blood sugar levels. Diabetes is becoming increasingly more common throughout the world, due to increased obesity - which can lead to metabolic syndrome or pre-diabetes leading to higher incidences of type 2 diabetes. Authors have identified 10 parameters that play an important role in diabetes and prepared a rich database of training data which served as the backbone of the prediction algorithm. Keeping in view this training data authors developed a system that uses the artificial neural networks algorithm to serve the purpose. These are capable of predicting new observations (on specific variables) from previous observations (on the same or other variables) after executing a process of so-called learning from existing training data (Haykin1998). The results indicate that the ANN is the best predictor with the accuracy of about $96 \%$. This system can be used to assist medical programs especially in geographically remote areas where expert human diagnosis not

possible with an advantage of minimal expenses and faster results.

Keywords: Artificial Intelligence, metabolic, Machine Learning, Diabetes, Artificial Neural Network, Medical Diagnosis.

\section{INTRODUCTION}

Diabetes is a disease that occurs when blood glucose, also called blood sugar, is too high. Blood glucose is main source of energy and comes from the food we eat. Insulin, a hormone made by the pancreas, helps glucose from food get into your cells to be used for energy. Sometime body doesn't make enough - or any-insulin or doesn't use insulin well. Glucose then stays in blood and doesn't reach cells. Diabetes can mainly be of 3 types: Type-1 diabetes, Type- 2 diabetes and Gestational diabetes. Type-1 diabetes results from non-production of insulin \& Type-2 diabetes results from development of resistance of insulin, as a result of which the insulin produced is not able to metabolize the sugar levels properly. Gestational diabetes occurs in pregnant women, who develop a high blood glucose level during pregnancy who never had any previous such history. It may be preceded by development of type-2 diabetes. As reported by WHO \& International Diabetic Federation in year 2010, the toll of diabetic patients was 285 million and this number is expected to grow to 483 million by 2030 . WHO estimates that between 2010 to 2030 there will be an increase of $69 \%$ in adult diabetic population in developing countries and $20 \%$ increase of the same in developed countries. India 
having 50.8 million patients of this disease leads the world \& is followed by China (43.2), United States (26.8). In 2010 diabetes caused 3.9 million deaths worldwide. The primary concern of AI in medicine is the construction of AI programs that can assert a medical doctor in performing expert diagnosis. These programs by making use of various computational sciences such as statistics and probability find out the hidden patterns from the training data and using these patterns they classify the test data into one the possible categories. The backbones of these AI programs are the various data sets prepared from various clinical cases which act as practical examples in training the system. The decision and recommendation prepared from these systems can be illustrated to the subjects after combining them with the experience of human expert.

\section{METHODOLOGY}

An artificial neural network (ANN) is a mathematical model which is inspired by the biological network of neurons. An ANN is a powerful data-modeling tool that is able to capture and represent and simulate complex relationships between inputs and outputs by performing multiple parallel computations. ANNs are computational analytic tools, modeled after the processes of "learning", in the cognitive system and the neurological functions of the brain. These are capable of predicting new observations (on specific variables) from previous observations (on the same or other variables) after executing a process of so-called learning from existing training data (Haykin 1998). ANN consists of a group of highly interconnected processing elements called "artificial neurons" and it processes information using a connectionist approach to computation. An ANN consists of a pool of processing units which communicate with each other by sending signals over a large number of weighted connections. ANN is an adaptive system that changes and adapts its structure during a learning phase. ANNs have been shown to perform more accurately as compared to its counterparts because of their ability to deal with incomplete and noisy data (Mahamud et al. 1999). One of the key elements of a neural network is its ability to learn. A neural network is complex adaptive system, that is, it can change its internal structure based on the information flowing through it. The predictive ability of an ANN can be improved by iterative learning algorithms. ANNs are now days widely being used in solving problems of type, "easyfor-a-human, difficult-for-a-machine", such as various problems in domain of pattern recognition, speech recognition, computer vision, and also in medical diagnosis. The ability of ANNs to recognize and classify various patterns accurately has attracted the attention of many researchers to solve problems from clinical domain. In last 20 years, ANN has been most popularly used AI technique in medical data mining (Ramesh et al. 2004). ANNs are typically characterized by three parameters: (1) Pattern of interconnection between different artificial neurons. The neurons are grouped into different layers, typically an input layer, one or more hidden layers, and an output layer. (2) The learning, which is achieved by repeatedly adjusting the numerical weights associated with the interconnecting edges between different artificial neurons. (3) Activation function that converts a neuron's weighted input to its output activation.

\section{PREVIOUS WORK}

It has been noted that the machine learning algorithms are increasingly being used in solving problems in Medical Domains such as in Oncology (Lisboa and Taktak 2006; Catto et al. 2003; Anagnostou et al. 2003; Bratko and Kononenko 1987), Urology (Anagnostou et al. 2003), Liver Pathology (Lesmo et al. 1983), Cardiology (Catlett 1991; Clark and Boswell 1991), Gynecology (Nunez 1990), Thyroid disorders (Hojker et al. 1998; Horn et al. 1985), and Perinatology (Kern et al. 1990). Numerous researchers have used AI and data mining-based algorithms to solve problems in the field of medicine. Chiu et al. (2008) studied the application of artificial neural networks in predicting the skeletal metastasis in patients suffering from prostate cancer. They analyzed whole body bone scintigraphies of patients with prostate cancer who underwent the technetium$99 \mathrm{~m}$ methylene diphosphate (tc-99 m MDP) for a period of five years. For the analysis, they used an artificial neural network having four layers of perceptrons which was trained with the data set of 111 prostate cancer patients. For evaluating the performance of ANN, receiver operating characteristics (ROC) analysis was used. The area under the ROC curve $(0.88 \pm 0.07)$ revealed excellent discriminatory power $(p \backslash 0.001)$ with the best simultaneous sensitivity $(87.5 \%)$ and specificity $(83.3$ $\%)$. Authors concluded that an ANN, which is based on limited clinical parameters, to be a promising method in forecasting of the skeletal metastasis. 


\section{PARAMETERS USED IN ESTIMATION}

Since India is having the highest Diabetic population in the world so it was easy to collect the data about the patients who suffered from this disease. After a detailed study, authors identified ten best physiological parameters for the study which were so chosen that the values for them could be easily determined and could be assigned discrete values, for the sake of maintaining consistency. Table-I summaries the parameters chosen and their allowed values. A dataset of 415 cases was prepared by collecting the data randomly from different sections of the society with an aim to have a variety in the dataset. To maintain accuracy and to avoid errors, considerable care was taken to ensure that the database had correct values.

Table-I: Various parameters used and their allowed values

\begin{tabular}{|c|c|c|}
\hline Parameter & Description & Allowed Values \\
\hline Age & Age of the subject & Discrete Integer Values \\
\hline Family History & $\begin{array}{l}\text { Whether any family member of the subject is suffering/ } \\
\text { was suffering from diabetes. }\end{array}$ & Yes or No \\
\hline Sex & Whether male or female & Male or Female \\
\hline Smoking & Whether the subject does smoking or not. & Yes or No \\
\hline Drinking & Whether the subject does drinking or not. & Yes or No \\
\hline Fatigue & Does a person feel tired after doing a little work? & Yes or No \\
\hline Thurst & $\begin{array}{l}\text { Whether the subject frequently feels a strong desire to } \\
\text { drink water. i.e how many times the subject drinks water. }\end{array}$ & Discrete Integer values \\
\hline $\begin{array}{l}\text { Frequency of } \\
\text { urination }\end{array}$ & How many times the subject passes urine in a day & Discrete Integer values \\
\hline Height & Height of the subject & $\begin{array}{l}\text { Discrete floating point } \\
\text { values }\end{array}$ \\
\hline Weight & Weight of the subject & $\begin{array}{l}\text { Discrete floating point } \\
\text { values }\end{array}$ \\
\hline
\end{tabular}

\section{IMPLEMENTATION}

In the present study, we have used the standard backpropagation-based multilayer perceptron (MLP) architecture of ANN. This architecture is most commonly used architecture for ANN in medical research. It consists of multiple layers of artificial neurons arranged in a directed graph, having each layer being fully connected to the next layer. All these are connected in a feed forward fashion with input layer connected to hidden layer and hidden layer connected to output layer. Backpropagation is a gradient descent technique to minimize the error criteria and it is simply a way to determine the error values in hidden layers. A hidden layer allows ANN to develop its representation of input-output mapping. In backpropagation, error data at the output layer are "back propagated" to earlier ones, allowing incoming weights to these layers to be updated and adjusted such that the error between the input and desired output is as least as possible. For processing the inputs at the artificial neurons, Sigmoid function was used as the activation function. Forrealization of desired ANN, we used the Neural Network Toolbox of Matlab 7.6.0. Figure 1(a) shows the topology for the neural network used in the study and Figure 1(b) shows the Matlab program in execution. Numbers of artificial neurons in the input, hidden, and output layers are 10, 20, and 1, respectively. To build the model, we repeated the training process up to one million times or under 0.0001 for the mean squared error in the model. The sigmoid function was employed, and the weight was decided randomly in the connections for the network. 


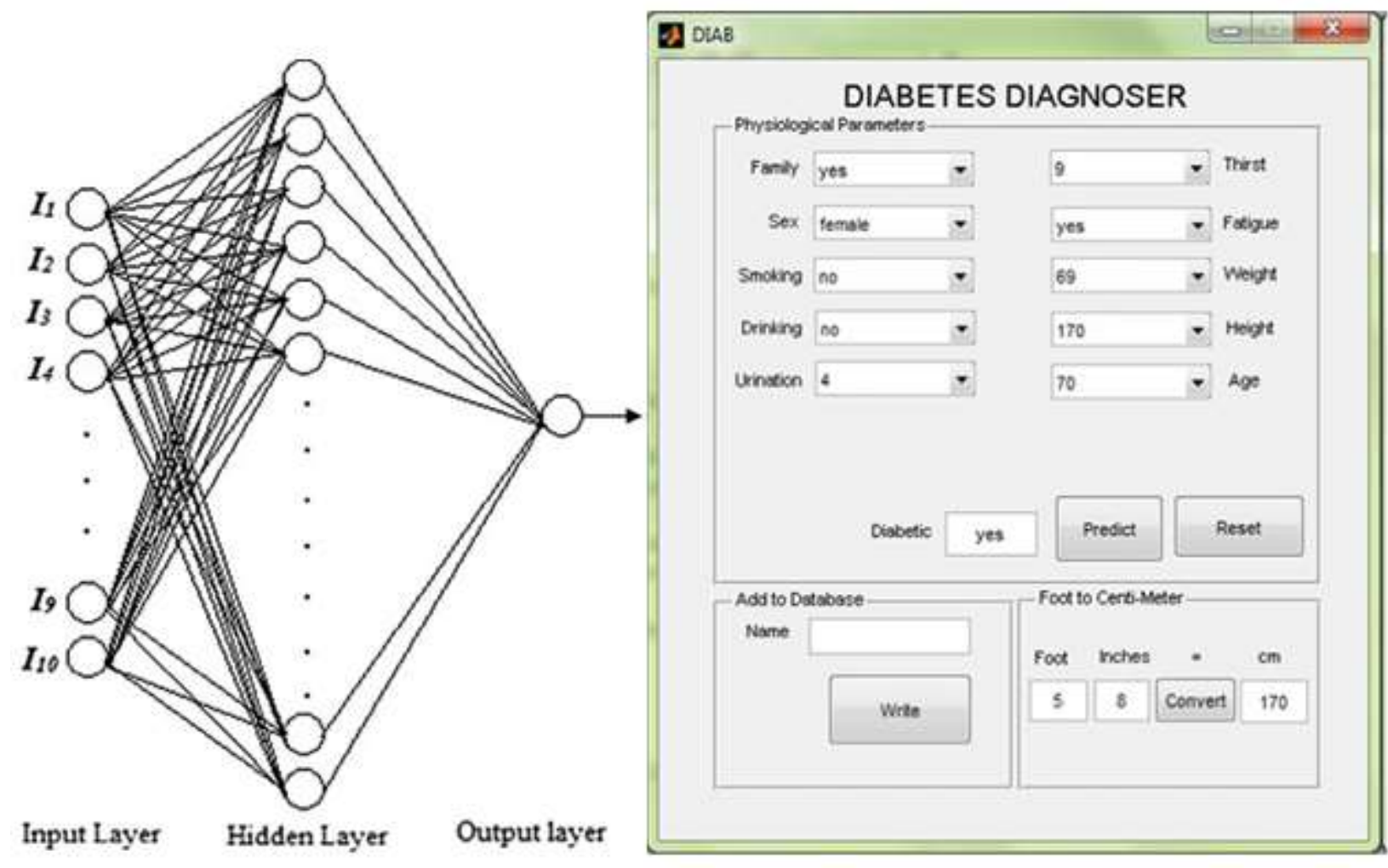

(a)

(b)

Fig 1(a) showing the topology for the neural network used in the study and Fig 1(b) showing the Matlab program in execution.

\section{CONCLUSION}

This artificial neural network based system is very useful for diagnosis of diabetes. The reliability of the system was evaluated by predicting new observations (on specific variables) from previous observations (on the same or other variables) after executing a process of so-called learning from existing training data. The results suggest that this system can perform good prediction with least error and finally this technique could be an important tool for supplementing the medical doctors in performing expert diagnosis. In this method the efficiency of Forecasting was found to be around $96 \%$. Its performance can be further improved by identifying \& incorporating various other parameters and increasing the size of training data.

\section{REFERENCE}

1) Anagnostou $T$, Remzi M, Lykourinas $M$, Djavan B (2003) Artificial neural networks for decisionmaking in urologiconcology. EurUrol 43(6):596603
2) Bharat Rao R, Bi J, Obuchowski N, Naidich D (2007) LungCAD: a clinically approved, machine learning system for lung cancer detection. In: International conference on knowledge discovery and data mining 2007, San Jose, California, USA, ACM 978-1-59593-609-7/07/0008

3) Bonham GS, Brock DB (1985) The relationship of diabetes with race, sex, and obesity. Am J ClinNutr 41:776-783

4) Bratko I, Kononenko I (1987) Learning rules from incomplete and noisy data. In: Phelip B (ed) Interactions in artificial intelligence and statistical methods. Technical Press, Hampshire

5) Caruana R, Niculescu-Mizil A (2006) An empirical comparison of supervised learning algorithms. In: ICML '06 Proceedings of the 23rd international conference on machine learning, pp $161-168$ 
6) Catlett J (1991) On changing continuous attributes into ordered discrete attributes. In: Proceedings of European working session on learning-91, Portugal, 4-6 Mar 1991, pp 164-178

7) Catto JWF, Linkens DA, Abbod MF, Chen M, Burton JL, Feeley KM, Hamdy FC (2003) Artificial intelligence in predicting bladder cancer outcome: a comparison of neuro-fuzzy modeling and artificial neural networks. Clin Cancer Res 9:4172

8) Chan JM, Rimm EB, Colditz GA, Stampfer MJ, Willett WC (1994) Obesity, fat distribution, and weight gain as risk factors for clinical diabetes in men. Diabetes Care 17(9):961-969
9) Chang C-L,HsuM-Y(2009) The study that applies artificial intelligence and logistic regression for assistance in differential diagnostic of pancreatic cancer. Expert SystAppl 36(7):10663-10672

10) Chiu J-S, Wang Y-F, Su Y-C,Wei L-H, Liao J-G, LiY-C (2008)Artificial neural network to predict skeletal metastasis in patients with prostate cancer. Springer science ?Business Media, May 2008

11) 11. Clark P, Boswell R (1991) Rule Induction with CN2: some recent improvements. In: Proceedings of European working session on learning-91, Portugal, Mar 1991, pp 151-163 